\title{
Distinguishing Ischemic from Non-Ischemic ST Changes: The Physionet/Computers in Cardiology Challenge 2003
}

\author{
GB Moody ${ }^{1}$, F Jager ${ }^{2}$ \\ ${ }^{1}$ Harvard-M.I.T. Division of Health Sciences and Technology, Cambridge, MA, USA \\ ${ }^{2}$ Faculty of Computer and Information Science, University of Ljubljana, Ljubljana, Slovenia
}

\begin{abstract}
Accurate diagnosis of myocardial ischemia during activities of daily living, if possible, would provide immediate and substantial benefits to affected subjects, by reducing the time needed to determine and evaluate therapeutic interventions intended to reduce or eliminate transient ischemia. Although ST changes provide a sensitive indicator of ischemia, a variety of non-ischemic mechanisms can also result in ST changes, so that ECG-based diagnosis of ischemia is regarded as non-specific and unreliable. It may be possible, by study of the dynamics of ST changes, to improve the specificity of ECG criteria consistent with ischemia. The fourth annual PhysioNet / Computers in Cardiology Challenge encouraged participants to develop and evaluate methods for distinguishing ischemic and nonischemic ST changes using a standard, meticulously annotated data set. The training data provided to participants, and the algorithms they developed, are available at http://www.physionet.org/challenge/2003/.
\end{abstract}

\section{Introduction}

For the fourth annual PhysioNet/Computers in Cardiology Challenge, we proposed a provocative question of considerable clinical interest: Is it possible to tell the difference between transient ST changes in the ECG that are due to myocardial ischemia, and those that are not?

For many years, a simple answer ("no") was considered to be the final word on this question. Myocardial ischemia results from insufficient oxygen delivery to the myocardium. To diagnose myocardial ischemia definitively, it is necessary to document that blood flow, blood oxygen saturation, or both have been compromised to an extent that the oxygen demands of the myocardium are not satisfied. These diagnostic criteria are typically established by imaging the coronary arteries. Since the ECG does not contain direct information about blood flow or oxygen saturation, it cannot be used to diagnose ischemia.

It may be possible, however, to establish associations between specific features of the ECG and myocardial is- chemia. One such association, between transient ischemia and changes in the ST segment, is highly sensitive but not specific. ST changes can also result from a wide variety of other causes, including changes in heart rate, conduction pattern, position of the subject, and noise in the ECG. Careful study of the ECG changes associated with these other causes of ST changes may make it possible to distinguish ischemic from non-ischemic changes[1], with immediate and substantial benefits for subjects whose ST changes occur in the context of activities of daily living, where other diagnostic methods are not practical.

Even in subjects who are known to have myocardial ischemia, ST changes are not considered a basis for definitive diagnosis of individual episodes of ischemia. In a subject with an old myocardial infarction, for example, the infarct may result in an ST segment with a persistent abnormal pattern (in the frame of reference of the heart). This fixed pattern appears to change with the subject's body position (upright, supine, etc.) because of movement of the ECG elecrodes relative to the heart. Thus many of those subjects who are most likely to experience ischemia are also among those most likely to have non-ischemic ST changes.

Therapeutic intervention to reduce or eliminate transient ischemic episodes can make a significant difference in quality of life for affected subjects, and may reduce mortality and morbidity in this population. Assessment of the effectiveness of therapy is substantially hindered by the lack of a reliable way of identifying ischemic episodes during activities of daily living, in which imaging studies are not possible. If it were possible to distinguish between ischemic and non-ischemic ST changes in ambulatory ECG recordings made during subjects' normal activities, the benefits would be immediate and substantial, in terms of a reduction in the time needed to determine and validate effective therapies, hence in the risk and pain experienced by the affected subjects.

As a public research resource, PhysioNet aims to provide the research community with freely available data and software that support and frequently bootstrap innovative studies in physiology, biomedical engineering, and medical physics. Consistent with this goal, PhysioNet and Computers in Cardiology have jointly sponsored an annual series of 
open, on-line challenges $[2,3,4]$ designed to stimulate rapid progress on interesting research and clinical questions. The current challenge is the fourth of this series.

\section{Organization of the challenge}

Participants in this year's challenge were asked to design and implement algorithms that can closely mimic the decisions made by human experts attempting to classify ST changes as ischemic or non-ischemic. Participants developed their algorithms using a, set of freely available data with expert-labeled ischemic and non-ischemic events; they then submitted their algorithms to PhysioNet, where the submitted code was compiled and evaluated using a separate data set.

\subsection{The reference data set}

This year's challenge encouraged participants to develop novel approaches to analysis of transient ST changes using the recently-completed Long-Term ST Database[5], a meticulously annotated collection of 86 recordings of 2- and 3-lead ECGs, each 20 to 24 hours long. Each ST change that meets criteria of clinical significance has been carefully studied by a team of expert annotators, who have drawn upon all available evidence to determine which of these events are consistent with a diagnosis of myocardial ischemia, and which are consistent with other causes. Half of this database has been contributed to PhysioNet and forms the learning set for the challenge (http://www.physionet.org/physiobank/database/ltstdb/); the remaining 43 recordings form the test set.

\subsection{ST event classifiers}

A short example program (available at http://www.physionet.org/challenge/2003/) was provided as a framework for the ST event classifiers to be entered in the challenge. The example contains an initialization function that sets any parameters required for a simulation, an analysis function that is invoked once per event, and a finalization function that is invoked after all events have been examined for a given recording. The rules of the challenge specified that participants write functions in standard (ANSI/ISO) $\mathrm{C}$ to replace these three functions in the example.

Entries were allowed to define additional functions, global and local variables; to use other functions from the ANSI/ISO C standard library and math library; and to create temporary files in the current directory (which, however, did not persist between runs). Entries were not permitted to modify the main (control) function provided in the example; to write to the standard output; to change the current directory; to start another program or process; or to include code or data that could not be made freely available after the conclusion of the challenge.

To enter the challenge, participants submitted their classifiers by email to PhysioNet, where the entries were compiled and used to classify the ST events in the test set. Each algorithm received a score determined by the number of correctly classified events, less the number of incorrectly classified events (those left unclassified did not affect the score). Scores were returned to participants by email. Participants were permitted to revise and resubmit their entries a limited number of times.

\subsection{Scoring}

The number of events per record varies considerably, from fewer than ten to several hundred. To avoid giving undue weight in the score to the handful of records that have a majority of the events, no more than 20 events from each record were used as a basis for scoring the entries; the others were not counted. The same set of events was used to score all entries.

\section{Results and Conclusions}

The final results are summarized in table 1. Although about 12 teams began work on this year's challenge, most of them found it too difficult. Only two groups completed the challenge. The top-scoring entry, from Philip Langley and colleagues, neatly separated the events that matched the expert annotators' objective criteria for events consistent with ischemia from the remaining events, obtaining a very high sensitivity and good positive predictivity. The group headed by Richard Povinelli had much better results with the training set, using a version of their algorithm coded using Matlab m-code, than they were able to obtain with either the training set or the test set using a reimplementation of that algorithm in $\mathrm{C}$ under time pressure.

\section{Acknowledgements}

PhysioNet/Computers in Cardiology Challenges are conducted using the facilities of PhysioNet, a public service of the Research Resource for Complex Physiologic Signals, which is supported by a grant from the National Center for Research Resources of the US National Institutes of Health (P41 RR13622). The authors thank the board and membership of Computers in Cardiology for their continuing and enthusiastic support of this series of challenges, and the participants who contributed their entries to PhysioNet for use and study by the research community at large. 
Table 1. Final results for Challenge 2003. Se: sensitivity (percentage of events consistent with ischemia that were classified as ischemic by the algorithm); +P: positive predictivity (percentage of events classified as ischemic by the algorithm that were consistent with ischemia).

\begin{tabular}{|c|c|}
\hline Performance & Entrant \\
\hline $\begin{array}{l}\text { Se: } 98.3 \% \\
+P: 82.8 \%\end{array}$ & $\begin{array}{l}\text { P Langley, EJ Bowers, N Brown, J Wilde, MJ Drinnan, J Allen, AJ Sims, A Murray } \\
\text { Medical Physics Department, Freeman Hospital } \\
\text { Newcastle upon Tyne, UK }\end{array}$ \\
\hline $\begin{array}{l}\text { Se: } 63.8 \% \\
+P: 48.2 \%\end{array}$ & $\begin{array}{l}\text { RJ Povinelli, MT Johnson, MW Zimmerman, KM Ropella } \\
\text { Marquette University } \\
\text { Milwaukee, Wisconsin, USA }\end{array}$ \\
\hline
\end{tabular}

\section{References}

[1] Jager F, Moody GB, Mark RG. Characterization of transient ischemic and non-ischemic ST segment changes. Computers in Cardiology 1995;721-724.

[2] Moody GB, Mark RG, Goldberger AL, Penzel T. Stimulating rapid research advances via focused competition: The Computers in Cardiology Challenge 2000. Computers in Cardiology 2000;27:207-210.

[3] Moody GB, Goldberger AL, McClennen S, Swiryn S. Predicting the onset of paroxysmal atrial fi brillation: The Computers in Cardiology Challenge 2001. Computers in Cardiology 2001;28:113-116.

[4] Moody GB. RR interval time series modeling: The PhysioNet / Computers in Cardiology Challenge 2002. Computers in Cardiology 2002;29:125-128.

[5] Jager F, Taddei A, Moody G, Emdin M, Antolic G, Dorn R, Smrdel A, Marchesi C, Mark R. Long-term ST database: a reference for the development and evaluation of automated ischaemia detectors and for the study of the dynamics of myocardial ischaemia. Medical and Biological Engineering and Computing 2003;41(2):172-182.

Address for correspondence:

George B. Moody

MIT Roon E25-505A, Cambridge, MA 02139 USA.

george@mit.edu 
